\title{
Investigation of the characteristics of the container for storage of radioactive waste of nuclear power plants with uranium-graphite reactors
}

\author{
Aleksey Ivshin $^{1}$, Alexandr Kalyutik ${ }^{1, *}$ and Anatolii. Blagoveshchenskii ${ }^{1}$ \\ ${ }^{1}$ Peter the Great St. Petersburg Polytechnic University, 29 Politekhnicheskaya street, 195251, Saint \\ Petersburg, Russia.
}

\begin{abstract}
The article presents the results of the study of neutron-physical characteristics of the container for storage of radioactive waste of nuclear power plants with uranium-graphite reactors. The interaction of gamma quanta (in the energy range from 0.1 to $2 \mathrm{MeV}$ ) with the structural materials of the container is simulated. The numerical values of the parameters determining the radiation characteristic of the container with the estimation of the calculation error are obtained. The following main characteristics of the container are determined: the attenuation coefficient of the equivalent dose, the numerical factor of gamma radiation accumulation. These characteristics can be used to justify the radiation safety of the container, in particular when selecting protection materials, as well as when building additional heterogeneous protection barriers.
\end{abstract}

\section{Introduction}

The development of nuclear power in Russia and abroad envisages the construction of new power units and the decommissioning of reactors that have exhausted their life, which will lead to an increase in the rate of accumulation of radioactive waste (RW) [1-9].

Radioactive waste requires special handling. Waste is placed in special containers, which must provide the necessary biological protection during transportation and temporary storage.

Loading a container with radioactive material requires that the equivalent dose rate on the surface of the packaging will be matched to the standardized values.

At the moment, a conservative approach to this task is being applied. The tolerances for the loading of radioactive waste (mass, volume, radionuclide composition, activity) for each container are established, under which the standardized parameters will meet the requirements of regulatory documents. The disadvantage of this method is that it is impossible to justify the safe handling of waste characteristics of which exceed the permissible limits. This requires additional complicated and time-consuming calculations

*Corresponding author: Kalyutik@yandex.ru 
with large time costs. The delay in decision-making related to the justification of safety will lead to an increase in the dose burden on personnel and financial losses.

In this study, it is proposed to use the engineering calculation of biological protection against extended gamma sources for a particular container. The proposed method is based on the use of universal tables to calculate the protection from photon radiation. Such data were obtained [10] and have practical application in determining the required thickness of protection only for point isotropic sources. In this paper, similar tables are calculated for an extended isotropic source, taking into account the geometry and materials of the container. For this purpose, the interactions of monoenergetic gamma quanta (in the energy range from 0.1 to $2 \mathrm{MeV}$ ) with structural materials of the container were considered. Radiation characteristics of the container are determined: the number of attenuation, the numerical factor of gamma radiation accumulation.

Based on the calculated radiation characteristics of this container, it is possible to create a library of constants and further supplement it with new information (the type of container and the corresponding values of the attenuation factor, accumulation factors). Such constants will allow designing an information system that automatically detects the amount of radioactive waste to be loaded into a container. The use of such a system will provide support for decision-making in the handling of radioactive materials.

\section{Formulation of the problem}

We studied the reduction of gamma-quanta radiation by wall of reinforced concrete container intended for the storage of radioactive contaminated graphite from the uraniumgraphite reactor. The graphite masonry contamination takes place due to neutron activation of graphite $\left({ }^{13} \mathrm{C}(\mathrm{n}\right.$, gamma $\left.){ }^{14} \mathrm{C}\right)$, impurities $(\mathrm{Cl}, \mathrm{Fe}, \mathrm{Co}, \mathrm{Zn}, \mathrm{Cs}, \mathrm{Eu}, \mathrm{Th}, \mathrm{U}$, etc.) [11], nuclides which settle on the masonry from damaged technological channel $(\mathrm{H}, \mathrm{O}, \mathrm{Fe}, \mathrm{Cr}$, particles of fuel, the fission products, etc.) [12-14], and also radioactive carbon from the reaction $\left({ }^{14} \mathrm{~N}(n, p){ }^{14} \mathrm{C}\right)[15]$. The radionuclide of carbon ${ }^{14} \mathrm{C}$ is a source of low-energy $\beta$ radiation $\left(\mathrm{E}_{\beta}=156 \mathrm{keV}\right)$, due to the self-absorption of carbon and the reduction of the wall of the container its contribution to the dose rate at the point of detection will be small. Thus, the main contribution will be formed at the expense of radionuclide which is deposited in the graphite stack as a result of the channel damage and activation of impurities. These are mainly gamma-radiation sources with the energy of gamma-quanta of up to $2 \mathrm{MeV}$.

Due to the fact that there are no the reliable data on the radioactive pollutions for graphite blocks, it is necessary to obtain a universal data able to simulate it. To determine the flux density of gamma-quanta on the surface of the container from any radionuclide, polluting graphite blocks, we with the help of the program MCC 3D [16] had simulated weakening of the gamma-irradiation by the wall of the container.

In this way we considered the reduction of gamma-quanta radiation with energies 0.1 ; $0.4 ; 0.7 ; 1.0 ; 1.1 ; 1.2 ; 1.3 ; 1.4 ; 1.5 ; 1.6 ; 1.7 ; 1.8 ; 1.9 \mathrm{MeV}$.

For this purpose a volumetric container model with graphite was created, and one should determine physical and chemical properties of the container, graphite source (polluted part of the graphite), and the detectors. The container is made of concrete with density $2.34 \mathrm{~g} / \mathrm{cm} 3$ in the form of a parallelepiped: its external dimensions are 1200x1200x1430 mm, internal dimensions are 960x960x1150 mm, wall thickness is 120 $\mathrm{mm}$. Within it the graphite with density $1.65 \mathrm{~g} / \mathrm{cm} 3$ is placed, which fills the entire volume of the container.

Graphite is presented in two graphite blocks, placed in the container. Dimensions of the first block are $959.8 \times 954.7 \times 1149.8 \mathrm{~mm}$, the dimensions of the second block (polluted part of graphite) are $959.8 \times 5 \times 1149.8 \mathrm{~mm}$. 
Detectors have the following characteristics: diameters are $40 \mathrm{~mm}$, they are located in two rows at distances of $1 \mathrm{~mm}$ and $1 \mathrm{~m}$ from the container surface, with the height step of $160 \mathrm{~mm}$.

Here is considered the limiting case, when the polluted layer of graphite is located along one of the inner wall. Flux of gamma-quanta is registered by means of 18 ideal gammadetectors, which are located at an altitude of the container along the outside of the walls and at a distance of $1 \mathrm{~m}$ from the container (fig. 1). For each energy gamma-quanta 109 stories are modelled.

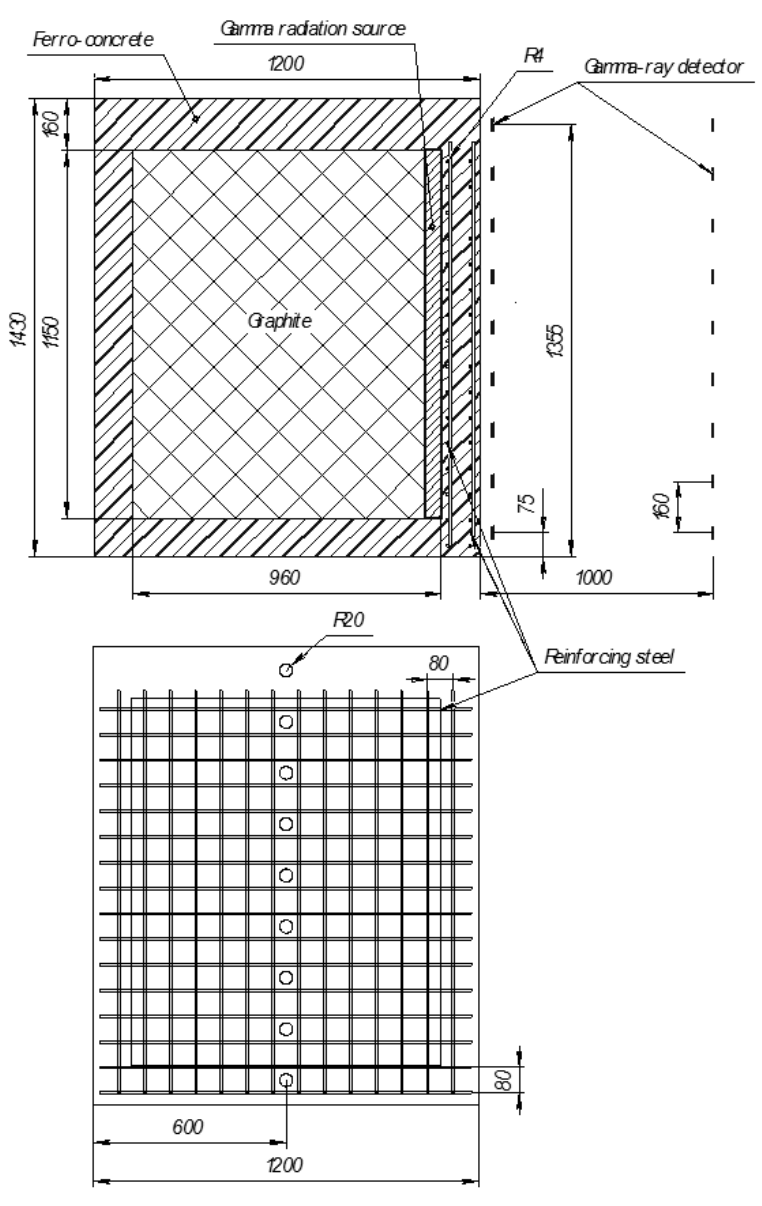

Fig. 1. The radioactive graphite placed in the protective container

\section{The equivalent dose}

To find the dose of gamma-radiation of continuous spectrum, one needs to divide the spectrum into the groups according to the particles' energies. The energies E $\gamma \mathrm{i}$ of the gamma-quanta are fixed and considered to be constant inside of each i-th group. The amount of gamma-quanta inside of each group are determined (fig. 2) as the area of the trapezoid (of the spectrum part). The desired equivalent dose is calculated in the form of the sum of the partial dose for each group. The equivalent dose is: 


$$
P=\sum_{i=1}^{m} N_{i} \cdot h\left(E_{\gamma i}\right) \cdot \Delta E_{\gamma i}
$$

where $\mathrm{m}$ is the number of energy groups; $\mathrm{Ni}$ is the number of gamma-quanta caught up in the $\mathrm{i}$-th group; $\mathrm{h}\left(\mathrm{E}_{\gamma \mathrm{i}}\right)$ is equivalent dose of gamma-radiation with energy [17]; $\Delta \mathrm{E}_{\gamma \mathrm{i}}$ is the width of the energy interval of the i-th group. The width of the energy interval is chosen constant: $\Delta E_{-} y_{i}=2 \mathrm{keV}$. The number of energy groups depends on the maximum energy of gamma-quanta of the ionizing radiation: $\mathrm{m}=\frac{E_{\max }}{\Delta E_{\gamma i}}$.

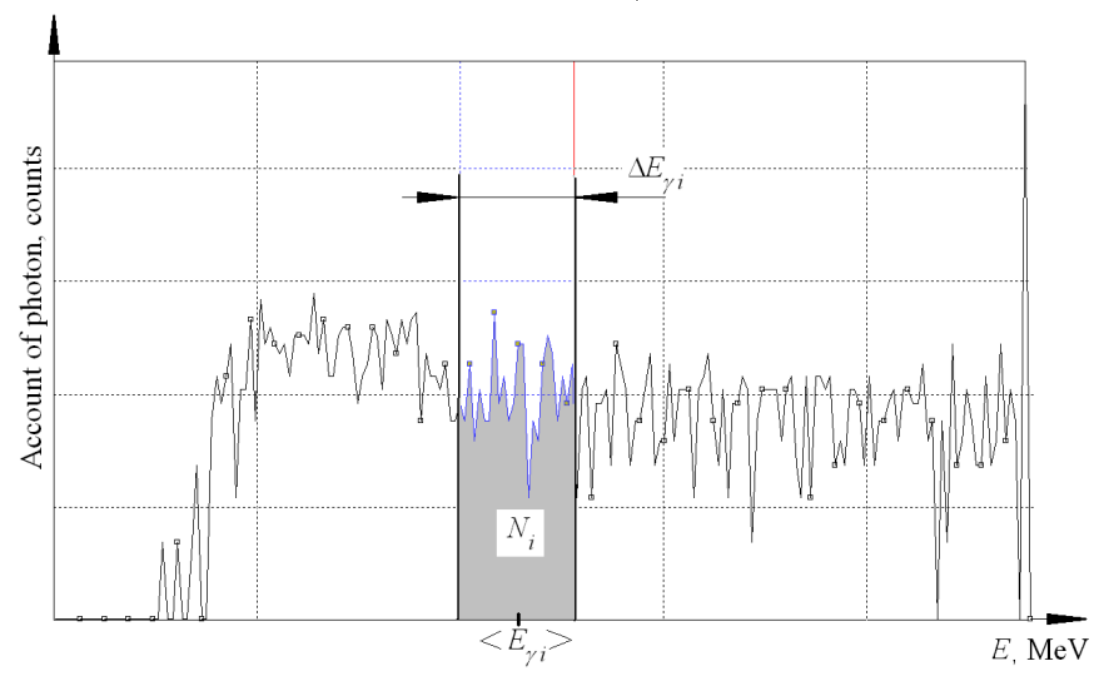

Fig. 2. The gamma-ray spectrum division into the energy groups

\section{The attenuation coefficient of gamma radiation}

The attenuation coefficient $K_{\text {att }}$ is the ratio of equivalent dose of the initial gamma radiation to the attenuated gamma radiation:

$$
K_{a t t}=\frac{P_{0}}{P_{d}}
$$

The relation between the attenuation coefficient and initial energy of gamma radiation is shown in fig. 3. Gamma radiation is considered on a surface of the container and at the $1 \mathrm{~m}$ distance from it, the detectors are located at the height of $\mathrm{H}=715 \mathrm{~mm}$. 


\section{EECE-2018}

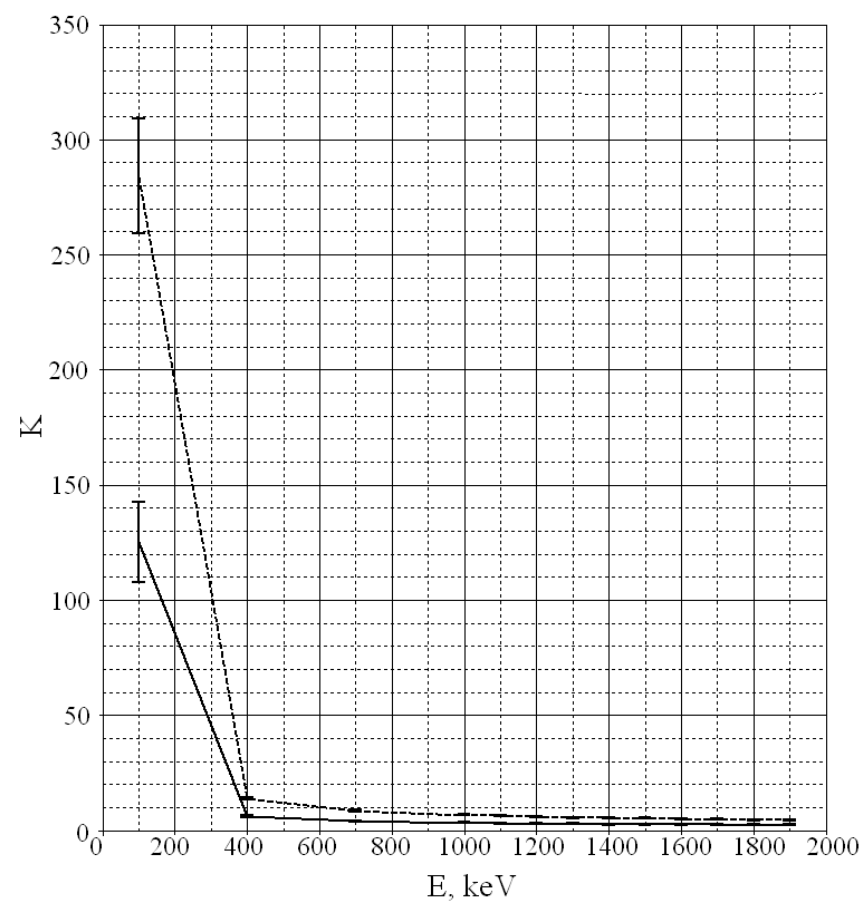

Fig. 3. Figure 3. Relation between the attenuation coefficient and initial energy of gamma radiation (- - - at the container surface - at a distance of $1 \mathrm{~m}$ from it)

The relative error of attenuation coefficient depends on number of the registered gamma quanta, the less is the number of registered accounts, the more the statistical error is.

The attenuation coefficient decreases with increase of initial energy of radiation (fig. 3 ).

According to standards of radiation safety, maximum permissible levels on a surface of packing and at distance of $1 \mathrm{~m}$ are regulated. Knowing the attenuation coefficients, it is possible to calculate maximum permissible capacities of an equivalent dose of initial radiation on the surface of the container $\left(H_{\text {perm } 1}\right)$ and at distance of $1 \mathrm{~m}\left(H_{\text {perm } 2}\right)$ which will satisfy the demanded level after attenuation by the container wall $\left(H_{d}\right)$. Maximum permissible capacity of an equivalent dose of the monoenergetic gamma radiation which does not interact with the protection is provided in the table:

$$
H_{\text {perm }}=K \times H_{d}
$$

Table 1. Maximum permissible capacity of an equivalent dose of the monoenergetic gamma radiation

\begin{tabular}{|l|l|l|}
\hline$E_{\gamma}, \mathrm{keV}$ & $H_{\text {perm } 1}, \mathrm{mSv} / \mathrm{h}$ & $H_{\text {perm } 2}, \mathrm{mSv} / \mathrm{h}$ \\
\hline 100 & $568 \pm 50$ & $12.5 \pm 1.7$ \\
\hline 400 & $27.7 \pm 1.3$ & $0.61 \pm 0.05$ \\
\hline 700 & $17.2 \pm 0.8$ & $0.39 \pm 0.03$ \\
\hline 1000 & $13.6 \pm 0.7$ & $0.32 \pm 0.03$ \\
\hline 1100 & $12.6 \pm 0.6$ & $0.3 \pm 0.03$ \\
\hline 1200 & $11.9 \pm 0.6$ & $0.28 \pm 0.03$ \\
\hline 1300 & $11.1 \pm 0.6$ & $0.27 \pm 0.02$ \\
\hline 1400 & $10.7 \pm 0.5$ & $0.27 \pm 0.02$ \\
\hline 1500 & $10.2 \pm 0.5$ & $0.25 \pm 0.02$ \\
\hline 1600 & $9.8 \pm 0.5$ & $0.24 \pm 0.02$ \\
\hline
\end{tabular}




\begin{tabular}{|l|l|l|}
\hline 1700 & $9.5 \pm 0.5$ & $0.24 \pm 0.02$ \\
\hline 1800 & $9.1 \pm 0.5$ & $0.23 \pm 0.02$ \\
\hline 1900 & $8.9 \pm 0.5$ & $0.23 \pm 0.02$ \\
\hline
\end{tabular}

Radioactive waste is usually a mixture of radioactive nuclides. Thus, the maximum permissible capacity of an equivalent dose of unreduced gamma radiation will be defined by a total contribution of each nuclide:

$$
H_{\text {perm }}=\sum_{(i)} H_{\text {permi }}=\sum_{(i)} K_{i} \cdot H_{d}
$$

\section{Numerical buildup factor of accumulation of ionization radiation}

After passing the protection the gamma radiation consists of scattered and primary gamma radiation. In some cases, contribution of the scattered radiation increases the dose of the reduced primary radiation by 1-2 orders of magnitude. This effect is accounted for by introducing a buildup factor $B$ in a law of reduction of the primary gamma rays:

$$
N_{d}=N_{0} \cdot \exp (-\mu \cdot d) \cdot B\left(E_{0}, \mu \cdot d, Z, r\right),
$$

where $\mu$ is the linear attenuation coefficient for photons of the energy $E_{0}, d$ is the linear thickness of the protection, $B$ is the buildup factor, $E_{0}$ is the gamma quantum energy, $Z$ is the atomic number of the material protection, $r$ is the distance from the radiation source to detector of gamma radiation.

Figure 4 shows the energy dependence of the buildup factor for protective container. Dose points are considered at the container surface and at the distance of $1 \mathrm{~m}$. 


\section{EECE-2018}

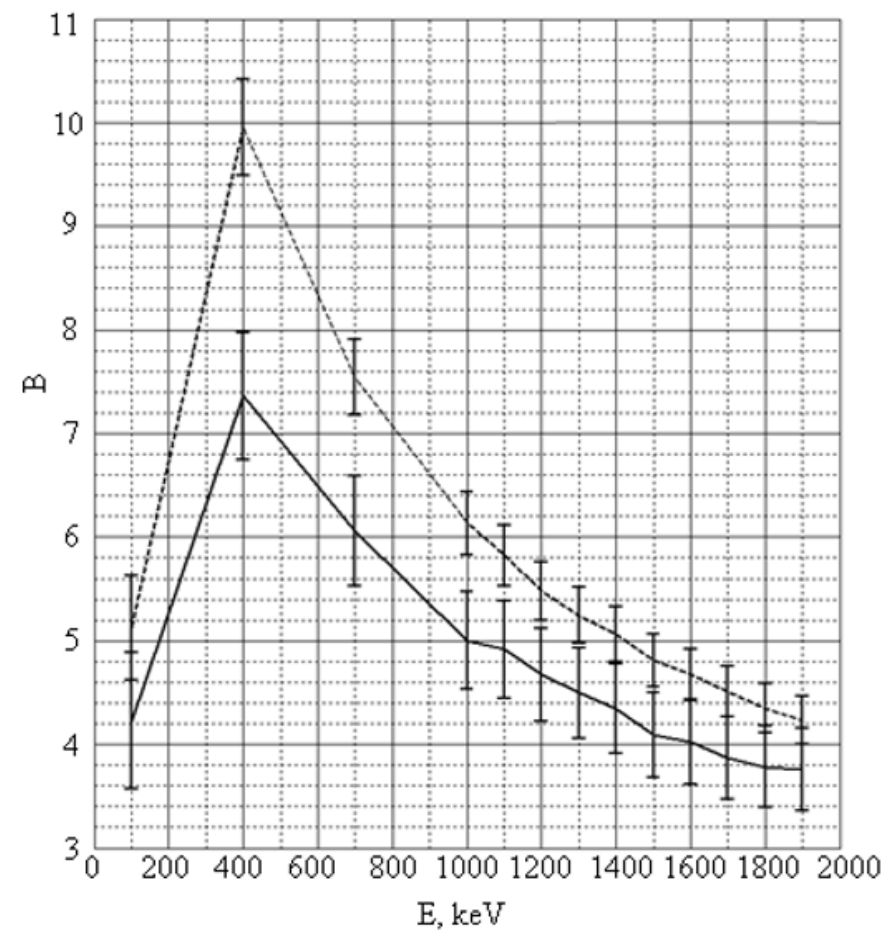

Fig. 4. Figure 4. The energy dependence of the buildup factor for protective container (- - - at the container surface, - at the distance of $1 \mathrm{~m}$ from container surface)

Relative error of determination the buildup factor depends on the number of registered particles. The less is the number of the registered particles the more is the relative error. The buildup factor increases with increase of the initial photon energy from 0.05 to 0.4 $\mathrm{MeV}$. The highest buildup factor is for $0.4 \mathrm{MeV}$ energy. Further, the buildup factor decreases with the increase of the initial energies of the gamma quanta from 0.4 to $2.0 \mathrm{MeV}$ (fig. 4). This results from the fact that cross section of the Compton scattering increases ( $\sigma_{C} \sim \frac{1}{E_{\gamma}}$ ) with decreasing of energies of the initial gamma-quanta, and this increases the contribution of scattered gamma rays. Cross section of the photoabsorption increases ( $\sigma_{a} \sim \frac{1}{E_{\gamma}^{3}}$ ) with decreasing of the initial photon energies, and this reduces quantity of gamma-quanta. The competition between these processes leads to appearance of peaks in gamma-quanta spectrum and in the buildup factors.

\section{Conclusions}

The reinforced concrete container designed to transportation and temporary storage of radioactive contaminated graphite blocks of channel-type reactor was investigated. The reduction of gamma radiation by the protective container was simulated. The radiation characteristics of the container with their relative errors were calculated.

The relative errors of equivalent dose, attenuation coefficient and buildup factor are almost equal and reach $10 \%$ for the scattered radiation and $5 \%$ for initial gamma radiation. 
The number of simulated events can be increased for reduction of relative errors of the results, and this requires increase in time of the account.

The attenuation coefficient of radiation decreases with increasing of gamma-ray energy. On the surface of the container it is reduced from 280 up to 4.5 , and at the distance of $1 \mathrm{~m}$ from the container surface its value is reduced from 130 up to 2.3.

The buildup factor increases with increasing of gamma-ray energy from 0.05 to 0.4 $\mathrm{MeV}$. The buildup factor has the maximum value at $0.4 \mathrm{MeV}$, and after that the buildup factor decreases with the increase of gamma-ray energy from 0.4 to $2.0 \mathrm{MeV}$.

Detailed investigation of the energy spectrum of gamma radiation outside of protection allows one to determine the contributions to dose for the scattered and no scattered radiations. These data can be used when selecting the material protection, and for the creation of additional barriers of heterogeneous protection.

\section{References}

1. Y.E.Karyakin, A.A. Pletnev, E.D. Fedorovich, E.D., Journal of Engineering Physics and Thermophysics 90(1), 80-87 (2017)

2. V. Maslikov, E. Negulyaeva, A. Cheremisin, A. Chusov, D. Molodtsov, A. Stroganov, Solid State PhenomenaVolume 871, 199-207 (2016)

3. N. Arefiev, M. Mikhalev, D. Zotov, K. Zotov, N. Vatin, O. Nikonova, O. Skvortsova, S. Pavlov, T. Chashina, T. Kuchurina, V. Terleev, V. Badenko, Y. Volkova, V. Salikov, K. Strelets, M. Petrochenko, A. Rechinsky, Procedia Engineering 117(1), 3238 (2015)

4. V.V. Davydov, V.I. Dudkin, E.N. Velichko, A.Yu. Karseev, Journal of Optical Technology (A Translation of Opticheskii Zhurnal) 82(3), 132-135 (2015)

5. A.S. Chugunov, A.V. Rumyantsev, V.A. Vinnitskiy, A.F. Nechaev, Izvestiya Wysshikh Uchebnykh Zawedeniy, Yadernaya Energetika 1, 119-127 (2015)

6. M.Y. Egorov, Atomic Energy 124(6), 403-407 (2018)

7. M.Y. Egorov, Atomic Energy 124(2), 139-142 (2018)

8. N.D. Agafonova, M.Y. Egorov, V.V. Sergeev, M.A. Gotovskii, P.A. Kruglikov, M.E. Lebedev, A.V. Sudakov, E.D. Fedorovich, B.S. Fokin, Atomic Energy 123(3), 154-158 (2018)

9. E.P. Obraztsov, A.N. Belikov, N.N. Menshikov, E.N. Popkov, E.N., ICAPP 2016 2, 1131-1140 (2016)

10. V.P. Mashkovich, A.V. Kudryavtsev, Protection from Ionizing Radiation: Handbook, 496 (1995)

11. A. Bushuev, J.M. Verzilov, V.N. Zubarev, I.M. Proshin, Measurement of trace elements in samples of graphite reactor by the neutron activation method. http://library.mephi.ru/data/scientific-sessions/2000/8/822.html

12. Y.A. Yegorov, Radiation safety in nuclear power plants, 153 (1986)

13. A. Bushuyev A., E.V. Petrova, A.F. Kojin, A.I. Shtyfurko,D.P. Masalit Investigation of radioactive contamination of the graphite samples from the reactor AM 5, 358-364 (2006)

14. A.A. Tsygankov, V.I. Tails, E.A. Mosquitoes, S.G. Kotlyarevksy, A.O. Pavlyuk, I.V. Shamanin, V.N. Nesterov, Bulletin of the Tomsk Polytechnic University 2, 94-98 (2007)

15. I.J. Vasilenko, V.A. Osipov, V.P. Rublevsky, Radiocarbon. Nature 12, 59-65 (1992) 
16. K.A. Bagaev, S.S. Kozlovsky, I.E. Novikov, Magazine «Anri» 4, 35-40 (2007)

17. A.A. Moiseev, V.I. Ivanov, Reference dosimetry and radiation hygiene, 296 (1984) 